\title{
The effect of standardised implantoplasty protocol on titanium surface roughness: an in-vitro study
}

\begin{abstract}
Andrew TAWSE-SMITH(a)
Akash KOTA ${ }^{(a)}$

Yathen JAYAWEERA(a)

Wendy Jansen van VUUREN ${ }^{(a)}$

Sunyoung $M^{(a)}$
\end{abstract}

(a) University of Otago, Sir John Walsh Research Institute, Faculty of Dentistry, Dunedin, New Zealand

Declaration of Interests: The authors certify that they have no commercial or associative interest that represents a conflict of interest in connection with the manuscript.

Corresponding Author:

Andrew Tawse-Smith

E-mail: andrew.tawse-smith@otago.ac.nz

DOI: 10.1590/1807-3107BOR-2016.vol30.0137

Submitted: Apr 28, 2016

Accepted for publication: Oct 21, 2016

Last revision: Oct 30, 2016

\begin{abstract}
To analyse the changes of surface characteristics of machined and moderately roughened titanium disks following a standardised implantoplasty protocol. Forty titanium discs (machined: $\mathrm{n}=20$; moderately roughened: $n=20$ ) were instrumented with one half of each disc maintained as the control (non-instrumented). The standardised implantoplasty protocol was carried out using a custom jig with the sequential change of burs: 1) Regular grit diamond [10s], 2) Super-fine grit diamond [10s], 3) Brownie ${ }^{\mathrm{TM}}$ silicone polisher [15s], 4) Greenie ${ }^{\mathrm{TM}}$ silicone polisher [15s]. Surface topography was analysed using confocal laser scanning microscopy (CLSM) and scanning electron microscopy (SEM). Energy dispersive x-ray spectroscopy (EDS) was used to measure the elemental profiles of each disc. Quantitative analysis showed similar changes in level of roughness between the machined and moderately roughened titanium discs. CLSM demonstrated an increased roughness (Ra and Sa values) after polishing with a regular grit diamond bur when compared to the uninstrumented surfaces. Although the roughness decreased after the further polishing with the super-fine grit diamond bur, subsequent instrumentation using silicon burs tended to increase the roughness, albeit being statistically insignificant. There was a residue of silicon particles despite the irrigation after each polishing stage. The proposed implantoplasty protocol did not achieve a sufficient level of smoothness on the machined or moderately roughened titanium surfaces when compared to the Ra threshold. Further research is recommended to test the efficacy of each bur on titanium surfaces with longer duration using actual oral implants to allow better comparison.
\end{abstract}

Keywords: Peri-implantitis; Dental Implants; Titanium; Microscopy, Confocal.

\section{Introduction}

Oral implants offer a successful long-term treatment option when replacing missing teeth. ${ }^{1,2}$ While its high predictability and long-term success rates have been well documented in the literature, it is not without ongoing maintenance or complication issues. One of the biological complication issues that can have detrimental impact on the long-term outcome of implant restorations includes peri-implantitis.

Peri-implantitis has been defined as an inflammatory disease of peri-implant supporting tissues associated with bacterial activity specifically, 
gram-negative rods such as Prevotella intermedia, Treponema denticola, Tanarella forsythia, Aggregatibacter actinomycetemcomitans and Porphyromonas gingivalis. ${ }^{3,4}$ It is characterised by bleeding on probing, increased probing depth, bone loss, and in several cases associated gingival recession. Links have been shown to the red complex microorganisms and gram-negative anaerobes who themselves are linked to the progression of periodontal disease as they have been found at the site of the affected areas. ${ }^{5}$ It has also been shown that patients who have a history of periodontal disease prior to implant treatment can experience higher rates of peri-implant diseases ${ }^{6,7}$ including those with other risk factors such as smoking or diabetes. ${ }^{8}$

The aim of treating peri-implantitis is to disrupt the biofilm, detoxify the implant surface and therefore improve surface biocompatibility. This has become more of challenge as rougher macro- and microscopic implant surfaces have been employed to improve the rate of osseointegration ${ }^{9,10}$ and maintaining plaque-free surfaces when the implants are exposed to the oral cavity is very difficult. Common treatment options for peri-implantitis include mechanical debridement, administration of local and systemic antibiotics, and complex surgical techniques. However, the surfaces must be detoxified using a chemical agent such as chlorhexidine, citric acid and hydrogen peroxide to further remove bacteria and concurrently eliminate or dilute the endotoxins formed by the previously colonised bacteria ${ }^{11,12}$ prior to any regenerative techniques are attempted often in combination of detoxification techniques. ${ }^{12}$ However, it has been shown that one-off mechanical treatment is inadequate in assuring the complete removal and prevention of recurrent disease.

Administration of systemic antibiotics have been shown to be effective for some peri-implant diseases in short-term studies, ${ }^{13}$ however, its long-term efficacy remains to be established. Treatment outcomes using other non-surgical therapies such as air abrasion, laser and chemotherapeutics have also been either inconclusive or ineffective to halt progression of disease. ${ }^{14,15}$ The use of ultrasonic scalers and/or manual carbon fibre curettes, are also inadequate for decontaminating implant surfaces with peri-implant pockets greater than or equal to $5 \mathrm{~mm}$.

Implantoplasty is an alternative option to improve the implant surface topography to reduce microbial adhesion and colonisation thus preventing re-infection of the site. ${ }^{7,17}$ This involves mechanically adjusting the exposed implant threads with rotary instruments to reduce the roughness of the surface and therefore minimise future plaque retention. ${ }^{17,18,19,20}$ Although it is well accepted that the affected rough implant surface must be altered to minimise the establishment of biofilm, there is no current consensus in the literature regarding a suitable polishing protocol.

The purpose of this in-vitro study was to assess the efficacy of polishing protocols in achieving a clinically viable threshold of titanium surface smoothness as well as evaluating any presence of foreign particles on the instrumented titanium surfaces at the completion of implantoplasty.

\section{Methodology}

\section{Titanium specimen}

Forty grade IV titanium disks with machined $(n=20)$ and moderately roughened $(n=20)$ surfaces were used. The moderately roughened disks had surface modification carried out by an ablative process, comparable to the currently available oral implants (Southern Implants Ltd, Irene, South Africa). Each titanium disk had identical dimensions (diameter $=9.0 \mathrm{~mm}$; thickness $=2.0 \mathrm{~mm}$ ) and was divided into two equal halves (surface area $=127.2 \mathrm{~mm}^{2}$ ) so that only the half of each disk was instrumented with the other side acting as a control. Sample size was calculated based on a power analysis using $G$ Power software ${ }^{21,22}$ for Mann-Whitney test. The power was set at 0.8 with an effect size of 1.2 and an alpha error probability of 0.05 . The power analysis showed that a total of 40 disks were required.

\section{Instrumentation protocol}

A custom-made device (Figure 1a) based on previous designs ${ }^{18,23}$ with further modifications which included a precision mobile disk stage and a pressure control lever was used. A handpiece (W\&H 
Synea Turbine TA-98 CLED, Ignaz-Glaser-Straße, Bürmoos, Austria) was secured in an adjustable jig leading to the mobile disk stage. ${ }^{18,22}$ In order to standardize the pressure applied during the timed polishing protocol (TPP), a force of $100 \mathrm{~N}$ was exerted and calibrated with an Orthodontics dial tension meter (Correx Haag-Streit Tension meter, Haag-Streit AG, Korniz, Switzerland) prior to each test (Figure 1b).
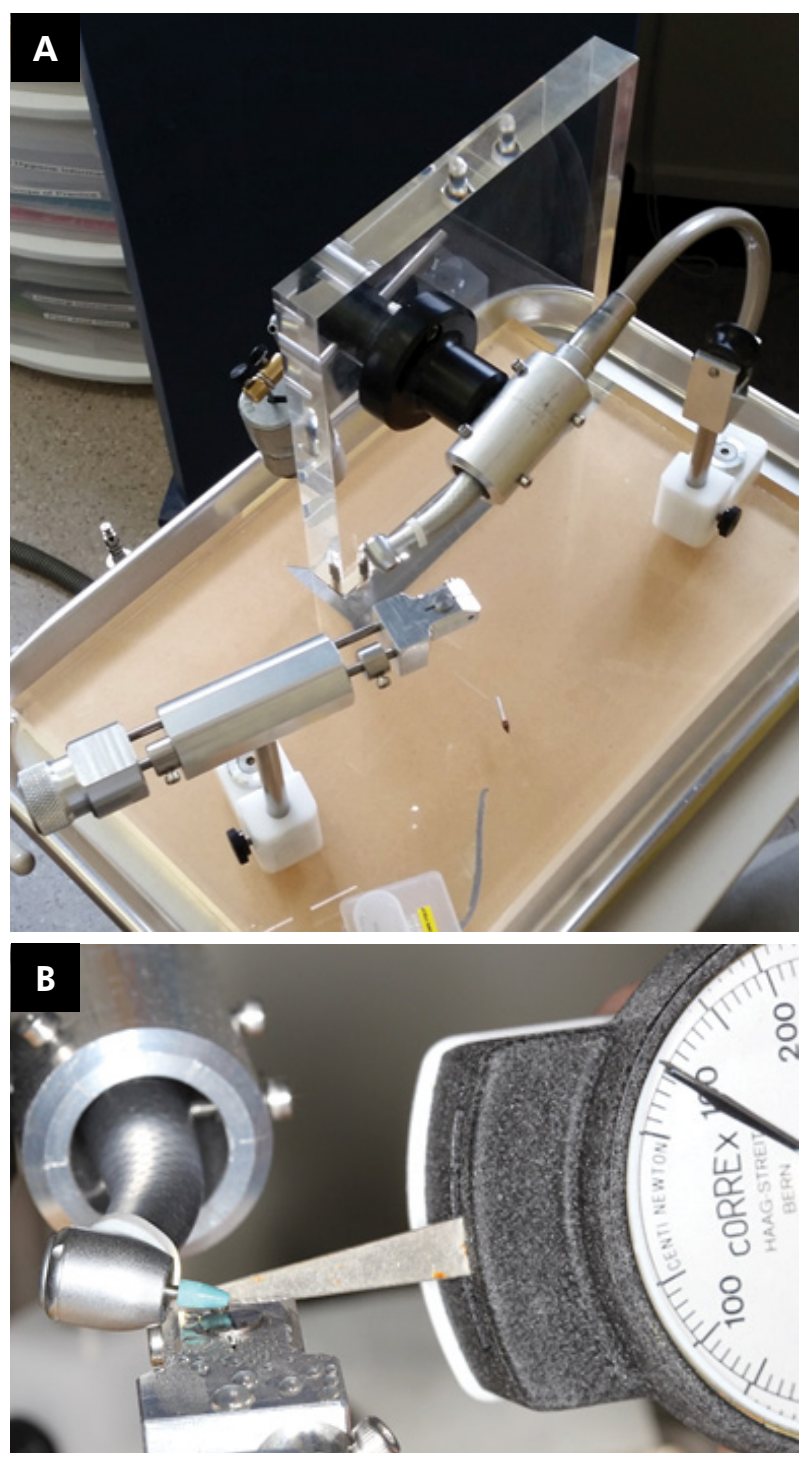

Figure 1. a) Custom-made instrumentation device standardises all instrumentation. Sliding disc stage, adjustable handpiece aligner and weight loading apparatus are featured. b) Calibration of the force exerted from the handpiece to the disk $(100 N)$ was conducted prior to each step.

\section{Burs}

Four different types of burs were used in the TPP: Shofu ${ }^{\mathrm{TM}}$ regular diamond (reference: 107RD), super-fine grit diamond (reference: SF107RD), Brownie ${ }^{\mathrm{TM}}$ (reference: PN0401) and Greenie ${ }^{\mathrm{TM}}$ (reference: PN040). Diamond burs were used 10 seconds each followed by silicone burs, which were applied for 15 seconds each (Figure 2). Burs were limited to a single use only and the titanium disks were stored securely in vials until microscopy analysis.

\section{Microscopy analysis}

Topographical analysis of the discs was carried out using confocal laser scanning microscopy (CSLM) (Zeiss LSM 510, Carl Zeiss Microscopy GmbH, Jena, Germany), scanning electron microscopy (SEM) (JEOL 6700F Field Emission SEM, Tokyo, Japan), and electron dispersive $x$-ray spectroscopy (EDS). SEM photomicrographs at various magnifications ( $25 x$ to $5000 x$ ) were acquired from all 40 discs using $5 \mathrm{kV}$ accelerated voltage.

When analysing with the CLSM, PlanApochroma x2/NA 0.6 objective was used with the confocal aperture adjusted to give an optical section depth of $3.0 \mu \mathrm{m}$ and operated in reflection mode with gain adjusted to avoid pixilation saturation. The limit of the $\mathrm{z}$-axis was determined during imaging. Images were taken at four sites per disc, two per instrumented and two per non-instrumented surface. Sites were chosen to avoid manufacturing features that could alter the surface roughness, such as digit and line scoring seen visually on the disc surface. The quantitative data was derived from qualitative surface topography created by ImageJ (Rasband, W.S., U.S. National Institutes of Health, Bethesda, USA, imagej.nih.gov/ij/, 1997-2012) and surface topography graphs were generated. Surface roughness measurements ( $\mathrm{Ra}$ and Sa values) were used to quantify the surface morphology of the discs. EDS (JEOL 2300) at an accelerating voltage of $25 \mathrm{kV}$ was used to derive the surface elemental composition of disc surfaces.

\section{Statistical analysis}

Mean values and standard deviations were derived from the raw data using a designated software 

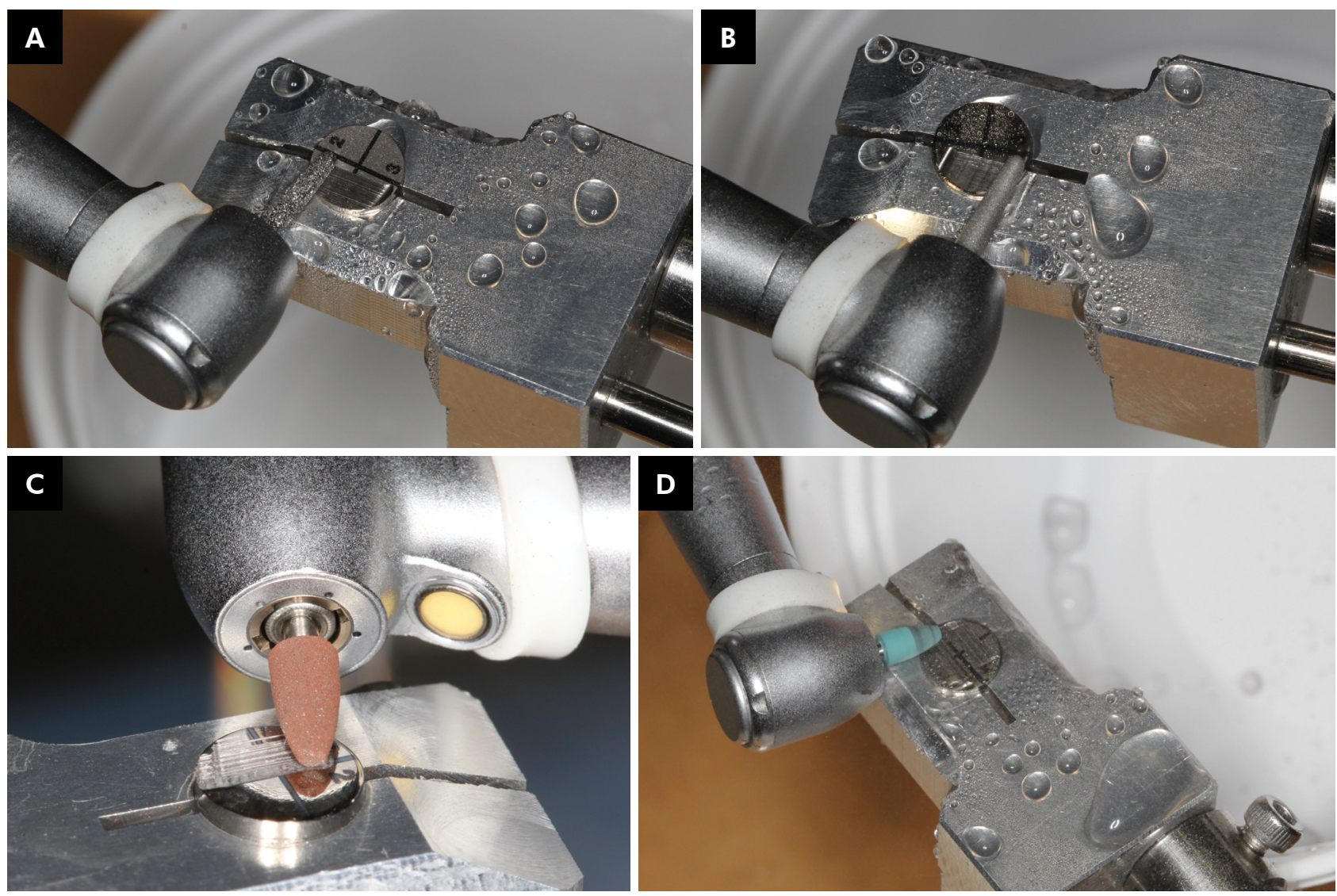

Figure 2. Burs used in implantoplasty protocol a) Regular-grit diamond. b) Super-fine grit diamond. c) Brownie ${ }^{\mathrm{TM}}$ silicone polishing point. d) Greenie ${ }^{\mathrm{TM}}$ silicone polishing point.

(Microsoft Excel. Redmond, Washington: Microsoft, 2013). Wilcoxon signed rank tests was conducted to detect differences between instrumented and control discs and Mann-Whitney U tests were done to compare the data between machined and moderately roughened discs. Kruskal-Wallis tests was also carried out to compare each instrumentation stage followed by a post hoc Dunn's test. Statistical significance was set at $\mathrm{p}<0.05$ (IBM SPSS Statistics version 2.0 IBM corp., Armonk, USA).

\section{Results}

\section{SEM}

SEM images of non-instrumented machined surfaces showed slight irregularities of concentric and isolated, with superficial linear surface marks which were characteristics of the milling process used (Figure 3a). Moderately roughened surfaces show a much more irregular abraded pattern, with pits and crevices due to the surface blasting with aluminium oxide particles (Figure $3 b$ ). The EDS profiles of non-instrumented surfaces consisted mainly of titanium (Figure 3c) whereas the moderately roughened surfaces (Figure $3 \mathrm{~d}$ ) showed traces of aluminium as expected from the grit-blasting process.

The regular grit diamond bur (Step 1) was effective in modifying the machined and moderately roughened titanium surface resulting in a wave-like ditching pattern with lateral striations throughout the instrumented area (Figure 4a). This polishing step resulted in a gross reduction of the original surfaces of the moderately roughened titanium discs (Figure 5a). Both surface types showed an irregular shelf-line pattern which was more evident under a higher magnification and a light irregular distribution of debris was also present on both instrumented and control surfaces. These changes 

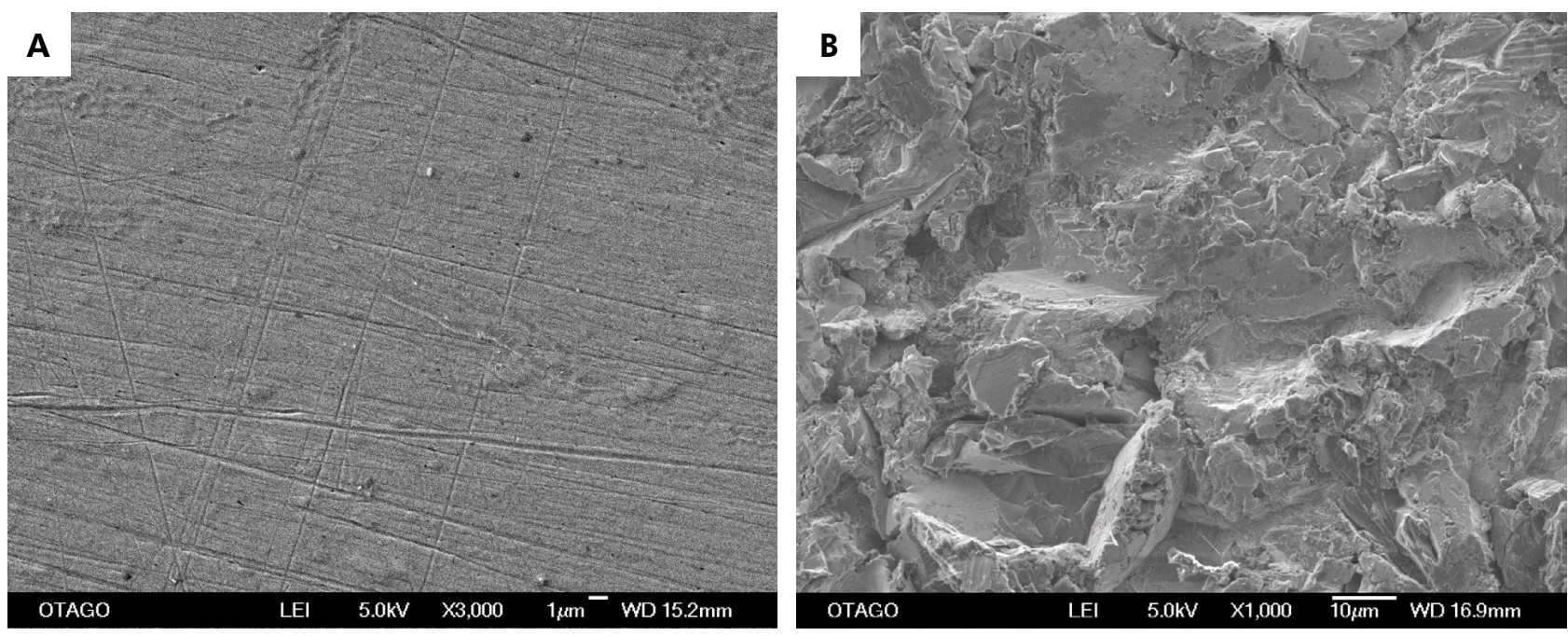

C
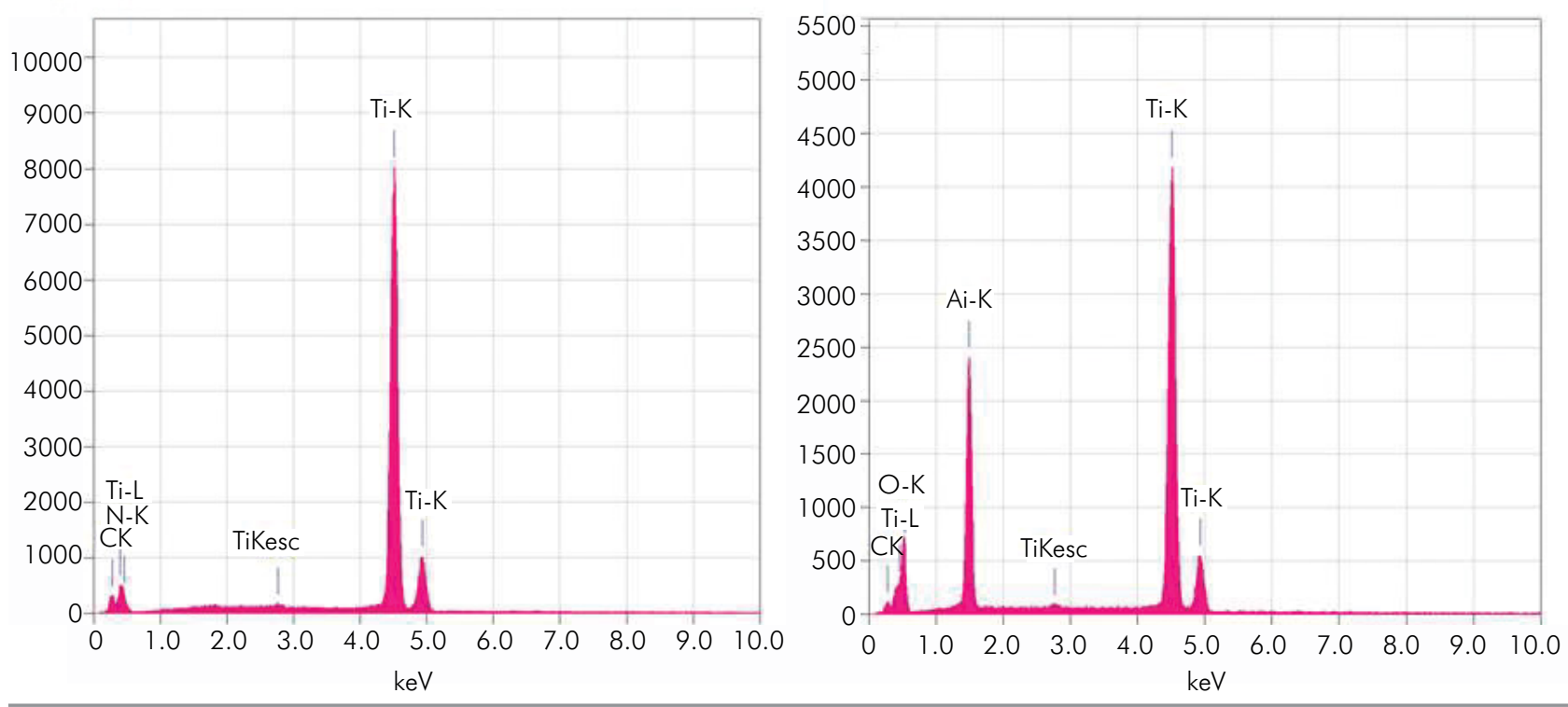

Figure 3. SEM images of non-instrumented surfaces of (a) Machined disk ( $\times 3000$ magnification) and (b) Moderately roughened (x1000 magnification) surfaces at respectively. EDS profiles of non-instrumented surfaces of (c) machined disk surface. (d) Moderately roughened disks, which show counts of aluminium.

were also apparent when the super-fine grit diamond bur was used in addition (Step 2). This additional polishing procedure reduced the width of the ditches and shelves formed previously resulting in a similar but much finer pattern (Figure $4 \mathrm{~b}$, $5 b)$. The proportion of random debris increased slightly for both instrumented and control surfaces (Figures $4 b, 5 b)$. Silicone polishing burs (Brownie ${ }^{\mathrm{TM}}$ ) were introduced at step resulting in a reduced depth of the ditches formed from previous steps, as confirmed by a smoother surface interpreted at higher magnification (Figure $4 c, 5 c$ ). The final polishing step included the Greenie ${ }^{\mathrm{TM}}$ silicone bur, which produced a further polished surface (Figure $4 d, 5 d$ ). Introduction of both types of silicone burs produced an increase in foreign debris with the last polishing protocol showing the most observed remaining foreign particles.

\section{Confocal Microscopy}

The control surfaces of both machined and moderately roughened titanium discs appeared relatively homogenous (Figure 6a, 6c). However, after the instrumentation, both groups appeared 

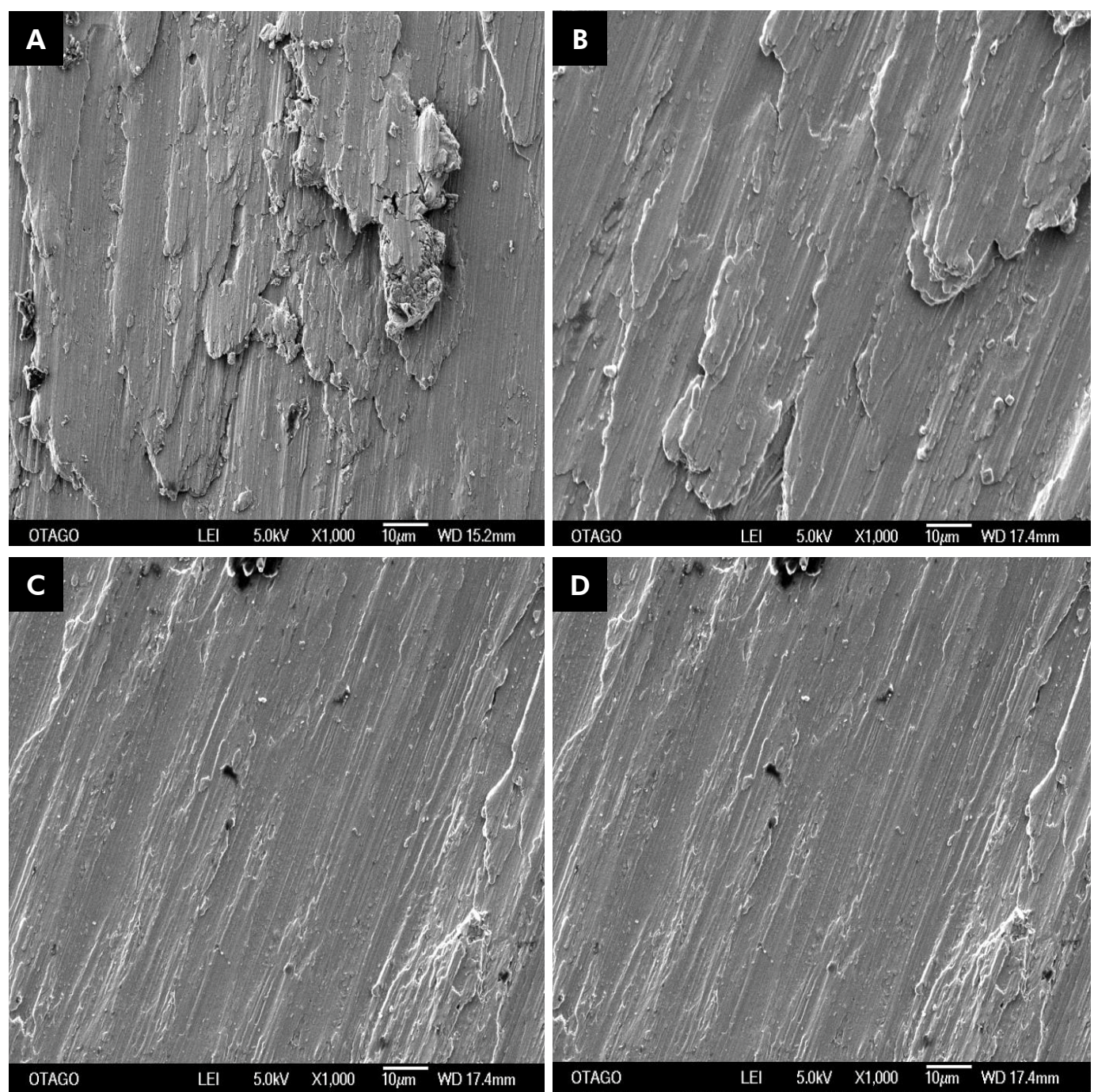

Figure 4. Machined disk surfaces following the corresponding steps of instrumentation (x1000 magnification) a) Step 1 of TPP b) Step 2 of TPP c) Step 3 of TPP d) Step 4 of TPP.

to have rougher surfaces while being reduced in surface height (Figure 6b, 6d). Each stage of polishing protocol showed a statistically significant difference in roughness $(\mathrm{Ra})$ compared to the corresponding controls (Table 1, 2). When comparing the mean roughness $(\mathrm{Ra})$ at each instrumentation step between the machined and moderately roughened titanium discs, there was no obvious difference except when the regular diamond bur (step 1) was used $(p=0.008)$ (Table 3).

\section{Discussion}

Several treatment options for treating peri-implantitis have been proposed in the literature with the main aims to control the infection, detoxify the implant and regenerate alveolar bone. ${ }^{25}$ As maintaining a plaque-free implant surfaces is necessary to achieve a positive treatment outcome, resective implantoplasty has the advantage of modifying the macroscopic topography of the titanium implants to reduce retention of plaque and debris. ${ }^{20}$ However, no clear guideline of armamentarium or clinical steps is yet to be suggested.

The implantoplasty protocol used in this in-vitro study has been modified since the original protocol ${ }^{18}$ by proving a larger titanium disc area to be instrumented and thus allowing for longer strokes of polishing. This avoided multiple cutting 

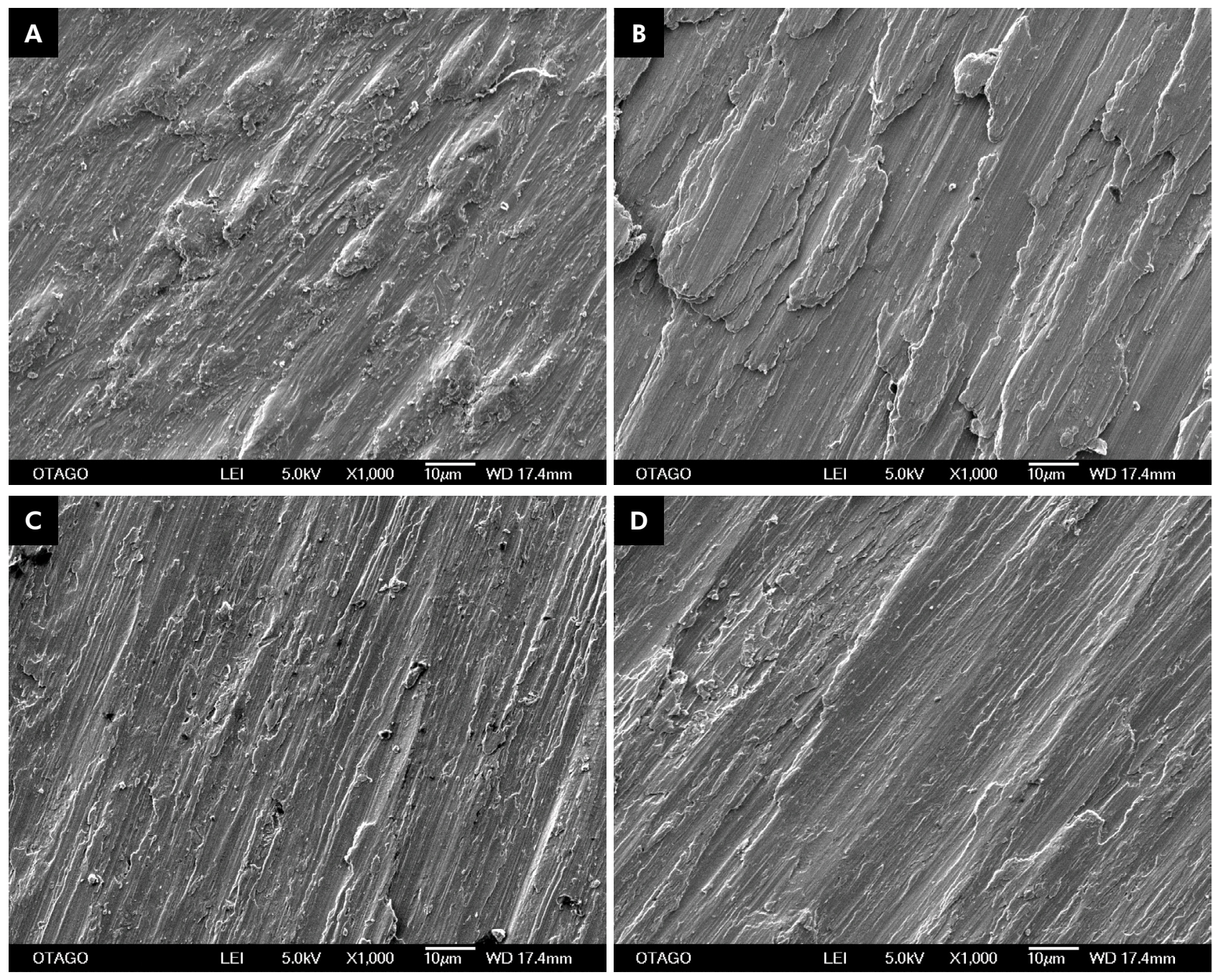

Figure 5. Moderately roughened disk surfaces following the corresponding steps of instrumentation (x1000 magnification) a) Step 1 of TPP b) Step 2 of TPP c) Step 3 of TPP d) Step 4 of TPP.

of the same surface which created a ditching effect. The TPPs followed a stepwise approach, where the titanium surface was initially recontoured and modified with regular and fine grit diamond burs, followed by the silicone burs as the final polishing protocol. The use of different types of burs were employed with the aim of reducing the surface roughness of titanium disks below a Ra threshold of $0.2 \mu \mathrm{m}$ to deter any biofilm formation. ${ }^{6,26,27}$ Other studies have included rough and smooth diamond burs as well as carbide and silicon burs with various sequences..$^{6,171,18,19}$ de Souza et al. ${ }^{19}$, demonstrated that three different rotational instruments: diamond, tungsten carbide and multilaminar burs resulted in similar surface roughness after performing implantoplasty procedures. However, the results from this study did not objectively standardised the time of instrumentation as it was determined by visual analysis of the operator only.

The proposed implantoplasty protocol resulted in a rougher surface after the instrumentation compared to the control. This indicates that the current polishing protocol alone is insufficient in decreasing the level of roughness to the desired Ra threshold of $0.2 \mu \mathrm{m}$. Instrumentation using only the regular diamond bur increased the roughness; however, there was no statistically significant difference in the changes of roughness level when 

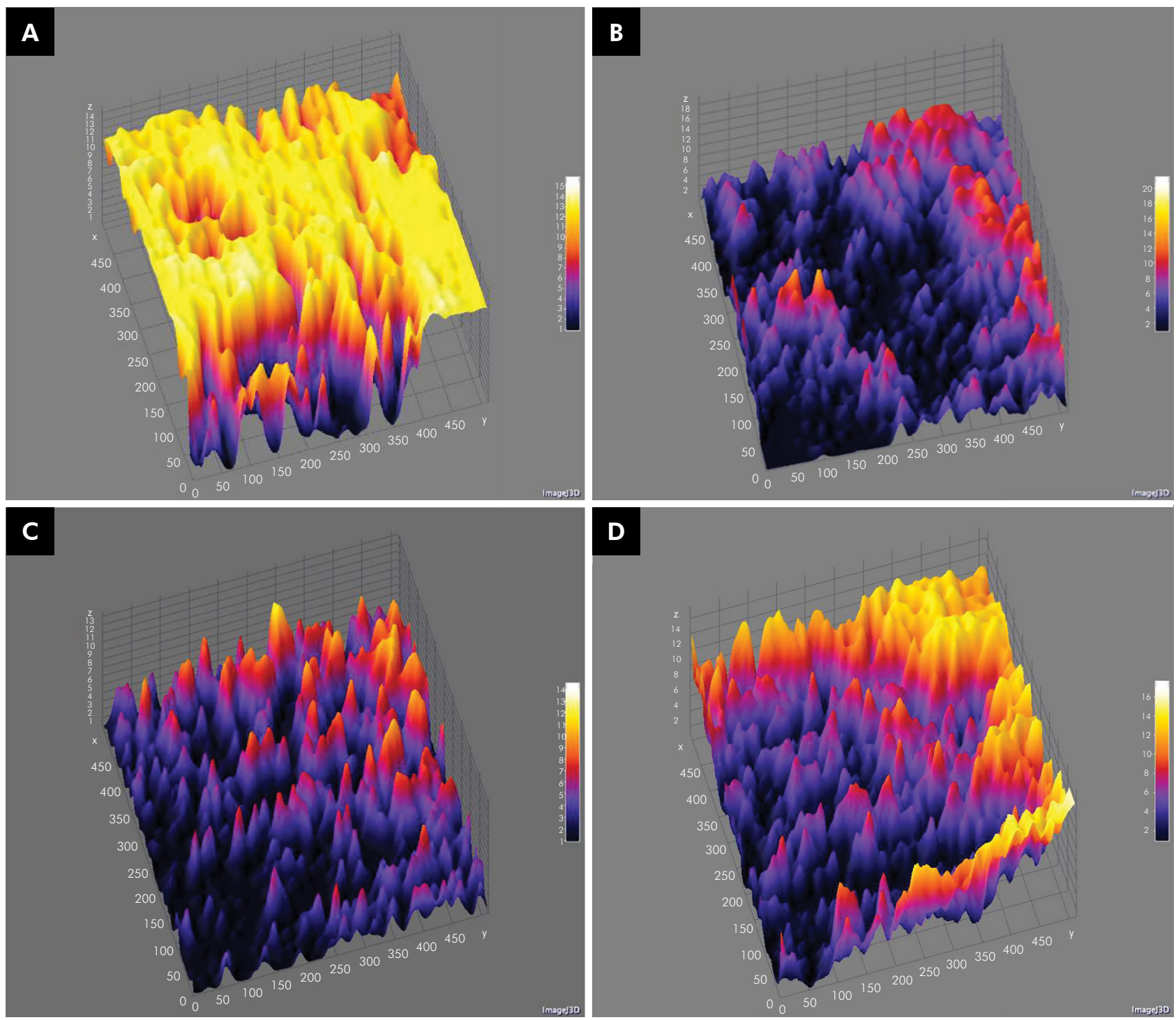

Figure 6. Surface topography of control surfaces and polished surfaces (after step 4). Graphs derived by ImageJ from confocal microscopy data. Peaks (yellow) and troughs (purple) indicative of the uniformity of the surface. a) Machined disk control surface. b) Machined disk polished surface. c) Moderately roughened disk control surface. d) Moderately roughened disk polished surface.

Table 1. Surface roughness averages and p-values for both instrumented and control surfaces of Moderately roughened Disks.

\begin{tabular}{|c|c|c|c|c|c|}
\hline \multirow{2}{*}{$\begin{array}{l}\text { Surface } \\
\text { Roughness }\end{array}$} & \multirow{2}{*}{$\begin{array}{c}\text { Implantoplasty } \\
\text { TPP }\end{array}$} & \multicolumn{4}{|c|}{ Implant surfaces } \\
\hline & & $\begin{array}{l}\text { Instrumented mean } \\
\text { (SD) }\end{array}$ & Control mean (SD) & Mean difference & $\mathrm{p}$-value \\
\hline \multirow{4}{*}{$\mathrm{Ra}$} & 1 & $4.93(0.45)$ & $1.68(0.47)$ & 3.25 & $0.043^{*}$ \\
\hline & 2 & $4.31(0.31)$ & $1.72(0.34)$ & 2.59 & $0.043^{*}$ \\
\hline & 3 & $4.46(0.23)$ & $2.04(0.61)$ & 2.42 & $0.043^{*}$ \\
\hline & 4 & $4.50(0.40)$ & $2.02(0.73)$ & 2.48 & $0.043^{*}$ \\
\hline
\end{tabular}

Ra: Surface roughness; TPP: time polishing protocol; *Statistical significance; Wilcoxon Signed Rank test. 
Table 2. Surface roughness averages and P-values for both instrumented and control surfaces of Machined Disks.

\begin{tabular}{|c|c|c|c|c|c|}
\hline \multirow{2}{*}{$\begin{array}{l}\text { Surface } \\
\text { Roughness }\end{array}$} & \multirow{2}{*}{$\begin{array}{c}\text { Implantoplasty } \\
\text { TPP }\end{array}$} & \multicolumn{4}{|c|}{ Machined surfaces } \\
\hline & & $\begin{array}{l}\text { Instrumented mean } \\
\text { (SD) }\end{array}$ & Control mean (SD) & Mean mifference & $\mathrm{p}$-value \\
\hline \multirow{4}{*}{$\mathrm{Ra}$} & 1 & $4.06(0.30)$ & $2.73(0.35)$ & 1.33 & $0.043^{*}$ \\
\hline & 2 & $3.93(0.46)$ & $2.70(0.32)$ & 1.23 & $0.043^{*}$ \\
\hline & 3 & $4.06(0.50)$ & $2.53(0.17)$ & 1.53 & $0.043^{*}$ \\
\hline & 4 & $4.00(0.52)$ & $3.09(0.29)$ & 0.91 & $0.043^{*}$ \\
\hline
\end{tabular}

Ra: Surface roughness; TPP: time polishing protocol; *Statistical significance; Wilcoxon Signed Rank test.

Table 3. Ra value comparison of instrumented machined and moderately roughened surfaces, at all steps.

\begin{tabular}{|c|c|c|c|c|}
\hline \multirow{2}{*}{ Implantoplasty } & \multicolumn{2}{|c|}{ Instrumented mean (SD) } & \multirow{2}{*}{ Mean difference } & \multirow{2}{*}{$\begin{array}{l}\text { P-value of machined c.f } \\
\text { moderately roughened }\end{array}$} \\
\hline & Machined & Moderately roughened & & \\
\hline Step 1 & $4.06(0.30)$ & $4.93(0.45)$ & 0.87 & $0.008^{*}$ \\
\hline Step 2 & $3.93(0.46)$ & $4.31(0.31)$ & 0.38 & 0.310 \\
\hline Step 3 & $4.06(0.50)$ & $4.46(0.23)$ & 0.40 & 0.056 \\
\hline Step 4 & $4.00(0.52)$ & $4.50(0.40)$ & 0.50 & 0.095 \\
\hline$p$-value comparing steps $1-4$ & 0.983 & 0.218 & - & - \\
\hline
\end{tabular}

*Statistical significance; Mann-Whitney U test.

further polishing was carried out with other burs. This was apparent for both the machined and moderately roughened titanium discs.

Visual interpretation of SEM and ImageJ generated surface topographies, indicated a pattern of reduction and polishing from Step 1 to 4 of the TPP. The wave-like pattern formed by regular-grit diamond instrumentation, was refined throughout the instrumentation steps as confirmed by the topographical maps, these align with results of Tawse-Smith et al. ${ }^{18}$

Another study ${ }^{28}$ which tested the polishing protocol of titanium abutments using fine diamond grit bur, silicon burs (Brownies and Greenies), cloth mops and a polishing compound showed that some areas might be roughened with diamond grit or carborundum, to the extent that in the final polishing steps these areas can be missed. However, it must be appreciated that polishing machined titanium surfaces without any thread features will be a different scenario to polishing an exposed oral implant. The current study indicated that there was 20 times higher Ra value of the instrumented titanium surface compared to the desirable Ra threshold which will be conducive to biofilm growth.
Systematic reviews have found the mechanical therapy produced variable results and rotary metal reduction overall, led to increased roughness. ${ }^{29}$ However, a recent study ${ }^{30}$ found that the best protocol was using diamond burs only in the order of descending grit, which performed better without the Greenie ${ }^{\mathrm{TM}}$ polishing point. There was also a statistically significant variation within the machined titanium disc (control) group, which may be explained by the accumulation of remaining foreign particles rather than the actual mechanical modification of the surface as confirmed by the SEM analysis.

Throughout the polishing protocol, the accumulation of surface debris increased despite irrigation after each instrumentation. The EDS detected traces of aluminium in the uninstrumented surfaces of the moderately roughened titanium discs due the aluminium oxide grit blasting used in the surface roughening process. However, it was noticed that after polishing the surface with the regular diamond bur, the aluminium particles were no longer detectable confirming the removal of at least the most superficial layer of the moderately roughened titanium discs. Instrumentation with the Brownie ${ }^{\mathrm{TM}}$ and Greenie $\mathrm{T}^{\mathrm{TM}}$ silicone burs introduced additional foreign particles detectable 
via EDS despite the use of water irrigation after each step. This is of significant clinical concern when carrying out implantoplasty as the instrumentation may introduce non-biocompatible foreign materials that remain in the treated areas, thus further complicating the treatment outcome. Others have also suggested minimising the usage of silicon points and using Arkansas stone to reduce any silicon debris remaining on the instrumented surfaces. ${ }^{30}$

As this in-vitro study simulated only the microscopic characteristics of the machined and moderately roughened implant surfaces without the macroscopic features of threads, the correlation of the results to the clinical setting may be limited. Further research in this field should include the introduction of different instruments/burs and different instrumentation duration including using the actual oral implants to allow more comparable results to an in vivo setting.

\section{References}

1. Ma S, Tawse-Smith A, Thomson WM, Payne AGT. Marginal bone loss with mandibular two-implant overdentures using different loading protocols and attachment systems: 10-year outcomes. Int J Prosthodont. 2010;23(4):321-32.

2. Simonis $P$, Dufour T, Tenenbaum H. Long-term implant survival and success: a 10-16-year follow-up of non-submerged dental implants. Clin Oral Implants Res. 2010;21:772-7. doi:10.1111/j.1600-0501.2010.01912.x

3. Heydenrijk K, Meijer HJ, Reijden WA, Raghoeber GM, Vissink A, Stegenga B. Microbiota around root-form endosseous implants: A review of the literature. J Prosthet Dent. 2003;89:517. doi:10.1016/S0022-3913(03)00272-5

4. Mombelli A, Müller N, Cionca N. The epidemiology of peri-implantitis. Clin Oral Implants Res. 2012;23(Suppl 6):67-76. doi:10.1111/j.1600-0501.2012.02541.x

5. Hultin M, Gustafsson A, Hallström H, Johansson LA, Ekfeldt A, Klinge B. Microbiological findings and host response in patients with peri-implantitis. Clin Oral Implants Res. 2002;13(4):349-58. doi:10.1034/j.1600-0501.2002.130402.x

6. Sharon E, Shapira L, Wilensky A, Abu-Hatoum R, Smidt A. Efficiency and thermal changes during implantoplasty in relation to bur type. Clin Implant Dent Relat Res. 2011;15(2):292-6. doi:10.1111/j.1708-8208.2011.00366.x

7. Ferreira SD, Silva GL, Cortelli JR, Costa JE, Costa FO. Prevalence and risk variables for peri-implant disease in Brazilian subjects. J Clin Periodontol. 2006;33(12):929-35. doi:10.1111/j.1600-051X.2006.01001.x

\section{Conclusion}

The proposed implantoplasty protocol did not achieve the level of smoothness on the machined or moderately roughened titanium surfaces when compared to the Ra of the control disks. Similar roughness levels were obtained for both surfaces after instrumentation. Further research is recommended to test the efficacy of each bur on titanium surfaces with longer duration using actual oral implants to allow better comparison.

\section{Acknowledgements}

The authors would like to thank Southern Implants (Irene, South Africa), Health Care essentials New Zealand and Shofu for their kind support with this study. The authors would also like to thank Ms Liz Girvan and Mr Andrew McNaughton for their guidance with electron microscopy and CLSM respectively, and Mr Andrew Gray for his expertise with the statistical analysis.

8. Heitz-Mayfield L. Peri-implant diseases: diagnosis and risk indicators. J Clin Periodontol. 2008;35(8 Suppl):292-304. doi:10.1111/j.1600-051X.2008.01275.x

9. Ektessabi A, Wennerberg A. Effects of surface topology on titanium release from implants into rabbit bone after 1-year follow up. Int J of PIXE. 1995;5(0203):145-52. doi:10.1142/S0129083595000174

10. Shalabi MM, Gortemaker A, Van't'Hof MA, Jansen JA, Creugers NH. Implant surface roughness and bone healing: a systematic review. J Dent Res. 2006;85(6):496-500. doi:10.1177/154405910608500603

11. Zablotsky MH, Diedrich DL, Meffert RM.

Detoxification of endotoxin-contaminated titanium and hydroxyapatite-coated surfaces utilizing various chemotherapeutic and mechanical modalities. Implant Dent. 1992;1(2):154-8. doi:10.1097/00008505-199205000-00009

12. Claffey N, Clarke E, Polyzois I, Renvert S. Surgical treatment of peri-implantitis. J Clin Periodontol. 2008;35(8 Suppl):316-32. doi:10.1111/j.1600-051X.2008.01277.x

13. Mombelli A, Lang NP. The diagnosis and treatment of peri-implantitis. Periodontol 2000. 1998;17(1):63-76. doi:10.1111/j.1600-0757.1998.tb00124.x

14. Haas R, Dörtbudak O, Mensdorff-Pouilly N, Mailath G. Elimination of bacteria on different implant surfaces through photosensitization and soft laser. an in vitro study. Clin Oral Implants Res. 1997;8(4):249-54. doi:10.1034/j.1600-0501.1997.080401.x 
15. Renvert S, Roos-Jansåker A, Claffey N. Non-surgical treatment of peri-implant mucositis and peri-implantitis: a literature review. J Clin Periodontol. 2008;35(8 Suppl):305-15. doi:10.1111/j.1600-051X.2008.01276.x

16. Karoussis IK, Salvi GE, Heitz-Mayfield LJ, Brägger U, Hämmerle $\mathrm{CH}$, Lang NP. Long-term implant prognosis in patients with and without a history of chronic periodontitis: a 10-year prospective cohort study of the ITI Dental Implant System. Clin Oral Implants Res. 2003;14(3):329-39. doi:10.1034/j.1600-0501.000.00934.x

17. Rimondini L, Cicognani Simoncini F, Carrassi A. Micro-morphometric assessment of titanium plasma-sprayed coating removal using burs for the treatment of peri-implant disease. Clin Oral Implants Res. 2000;11(12):129-38. doi:10.1034/j.1600-0501.2000.110205.x

18. Tawse-Smith A, Ma S, Wu H, Yeah A. Changes in surface characteristics of moderately roughened grade IV titanium disk following a standardised implantoplasty technique: an in-vitro study. Braz J Periodontol. 2015;25:22-33.

19. Souza Júnior JM, Oliveira de Souza JG, Pereira Neto AL, Iaculli F, Piattelli A, Bianchini MA. Analysis of effectiveness of different rotational instruments in implantoplasty: an in vitro study. Implant Dent. 2016;25(3):341-7. doi:10.1097/ID.0000000000000381

20. Gehrke SA, Aramburú Júnior JS, Dedavid BA, Shibli JA. Analysis of implant strength after implantoplasty in three implant-abutment connection designs: an in vitro study. Int J Oral Maxillofac Implants. 2016;31(3):e65-70. doi:10.11607/jomi.4399

21. Faul F, Erdfelder E, Lang AG, Buchner A. G*Power 3: A flexible statistical power analysis program for the social, behavioral, and biomedical sciences. Behav Res Methods. 2007:39(2);175-91. doi:10.3758/BF03193146

22. Siegel S, Fraunhofer J. Cutting efficiency of three diamond bur grit sizes. J Am Dent Assoc. 2000;131(12):1706-10. doi:10.14219/jada.archive.2000.0116
23. Chang CW, Waddell JN, Lyons KM, Swain MV. Cracking of porcelain surfaces arising from abrasive grinding with a dental air turbine. J Prosthodont. 2011;20(8):613-20. doi:10.1111/j.1532-849X.2011.00760.x

24. Faul F, Erdfelder E, Buchner A, Lang AG. Statistical power analyses using $G^{*}$ Power 3.1: Tests for correlation and regression analyses. Behavior Research Methods. 2009;41(4):1149-60. doi:10.3758/BRM.41.4.1149

25. Esposito M, Grusovin MG, Worthington HV. Treatment of peri-implantitis: what interventions are effective? A Cochrane systematic review. Eur J Oral Implantol 2012;5(Suppl S1):21-41.

26. Teughels W, Van Assche N, Sliepen I, Quirynen M. Effect of material characteristics and/or surface topography on biofilm development. Clin Oral Implants Res. 2006;17(Suppl 2):68-81. doi:10.1111/j.1600-0501.2006.01353.x

27. Bollen CM, Papaioanno W, Van Eldere J, Schepers E, Quirynen M, van Steenberghe D. The influence of abutment surface roughness on plaque accumulation and peri-implant mucositis. Clinical Oral Implants Res. 1996;7(3):201-11. doi:10.1034/j.1600-0501.1996.070302.x

28. Barbour ME, O'Sullivan DJ, Jenkinson HF, Jagger DC. The effects of polishing methods on surface morphology, roughness and bacterial colonisation of titanium abutments. J Mater Sci Mater Med. 2007;18(7):1439-47. doi:10.1007/s10856-007-0141-2

29. Louropoulou A, Slot DE, Van der Weijden FA. Titanium surface alterations following the use of different mechanical instruments: a systematic review. Clinical Oral Implants Res. 2011;23(6):643-58. doi:10.1111/j.1600-0501.2011.02208.x

30. Ramel C, Lüssi A, Özcan M, Jung RE, Hämmerle CH, Thoma DS. Surface roughness of dental implants and treatment time using six different implantoplasty procedures. Clin Oral Implants Res. 2015;27(7):776-81. doi:10.1111/clr.12682 